\title{
Erratum to "Beneficial Effects of Ethyl Pyruvate through Inhibiting High Mobility Group Box 1 Expression and TLR4/NF- $\kappa$ B Pathway after Traumatic Brain Injury in the Rat"
}

\author{
Xingfen Su, Handong Wang, Jinbing Zhao, Hao Pan, and Lei Mao \\ Department of Neurosurgery, Jinling Hospital, School of Medicine, Nanjing University, 305 East Zhongshan Road, Jiangsu Province, \\ Nanjing 210002, China \\ Correspondence should be addressed to Handong Wang, hdwang_nj@yahoo.com.cn \\ Received 28 May 2012; Accepted 4 July 2012 \\ Copyright (c) 2012 Xingfen Su et al. This is an open access article distributed under the Creative Commons Attribution License, \\ which permits unrestricted use, distribution, and reproduction in any medium, provided the original work is properly cited.
}

There is an error on Figure 9(c) in the original paper. The exact Figure 9 in the original paper has to be corrected as Figure 1 in this paper. 


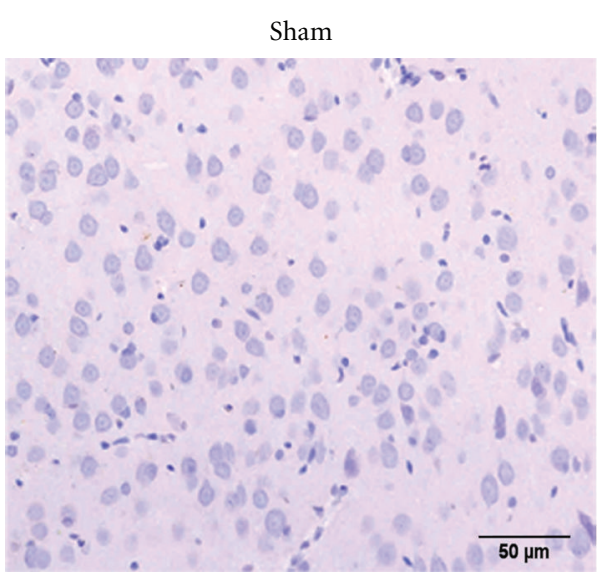

(a)

TBI + EP

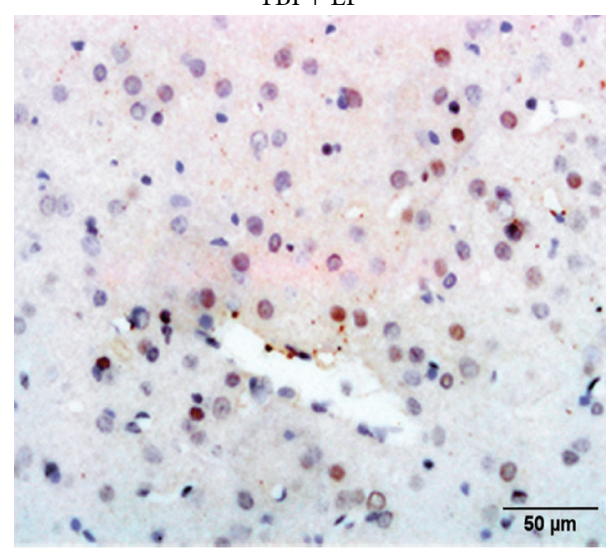

(c)

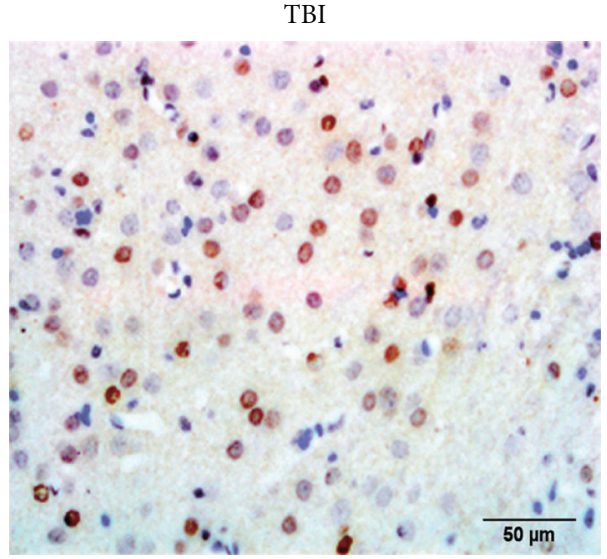

(b)

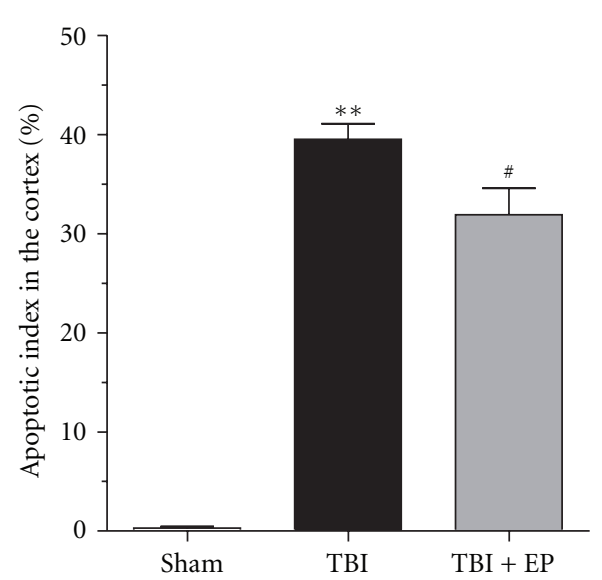

(d)

FIGURE 1 


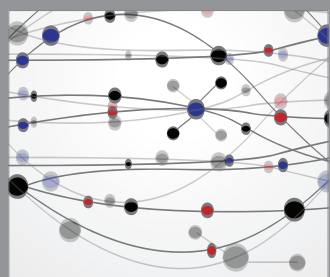

The Scientific World Journal
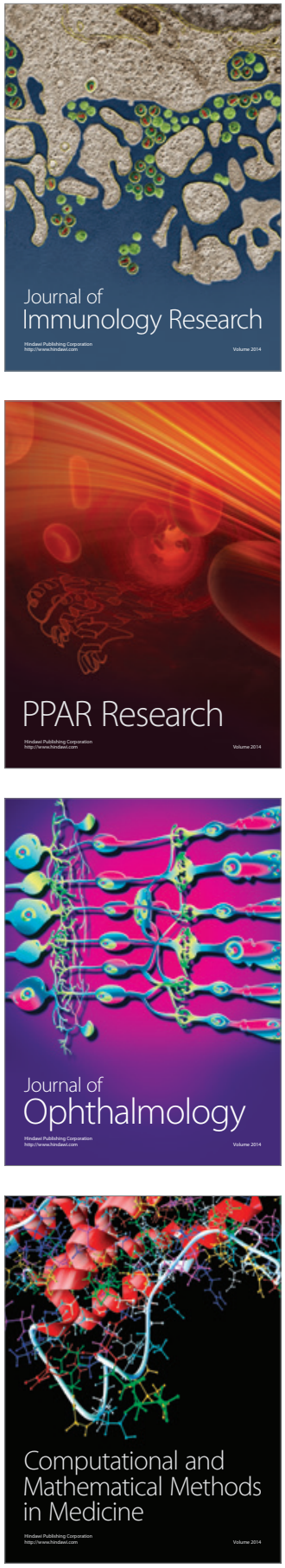

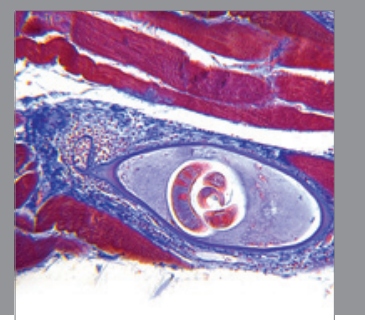

Gastroenterology

Research and Practice
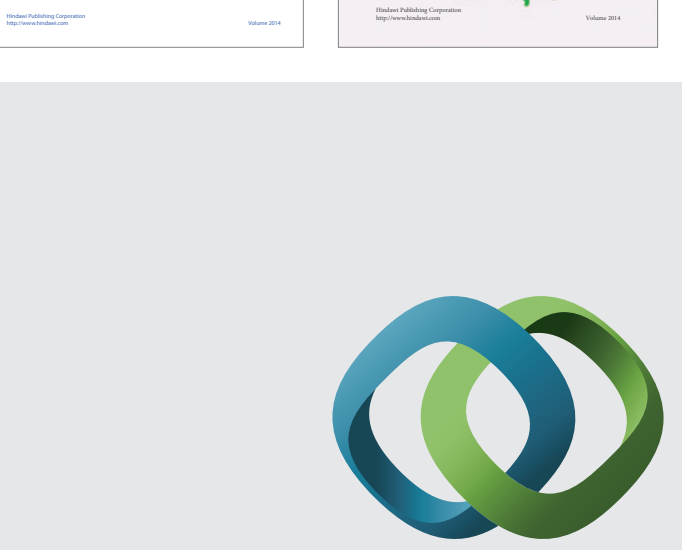

\section{Hindawi}

Submit your manuscripts at

http://www.hindawi.com
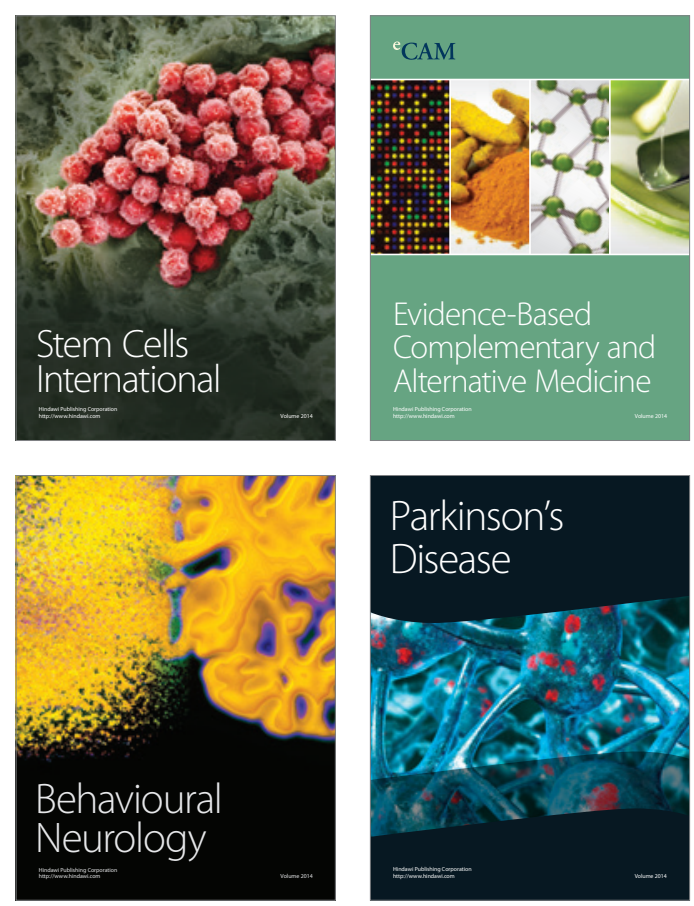

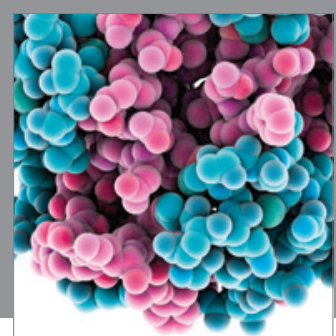

Journal of
Diabetes Research

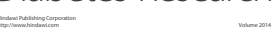

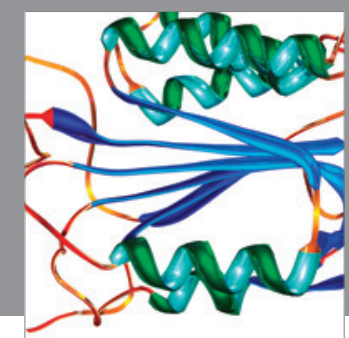

Disease Markers
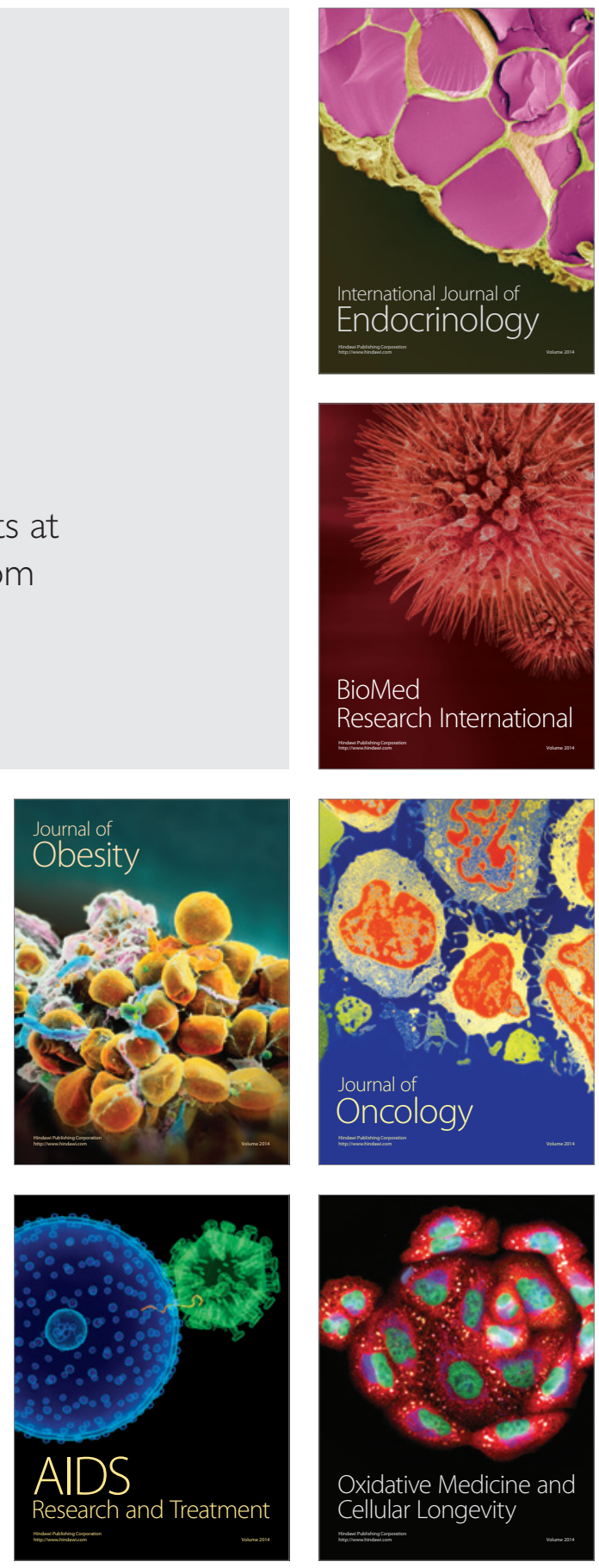